\title{
Visualizing the Distribution and Stoichiometry of Growth Factor Receptors in Intact Cells in Liquid Phase with Correlative Fluorescence and Scanning Transmission Electron Microscopy
}

\author{
Diana B. Peckys ${ }^{1}$, Justus Hermannsdörfer ${ }^{1}$, Verena Tinnemann ${ }^{1}$, Ulrike Korf $^{2}$ and Niels de Jonge ${ }^{1,3}$ \\ 1. INM - Leibniz Institute for New Materials, 66123 Saarbrücken, Germany \\ ${ }^{2}$ Division of Molecular Genome Analysis, German Cancer Research Center, 69120 Heidelberg, \\ Germany \\ ${ }^{3}$ Department of Physics, University of Saarland, 66123 Saarbrücken, Germany
}

The epidermal growth factor receptor (EGFR) family consists of four similar members that play important roles in various cellular processes such as cell proliferation and survival. Over-expression of these proteins, especially EGFR and HER2, is involved in many types of cancer. After activation by its ligand EGF, activated EGFR proteins can form homodimers, thus activating specific signaling pathways for cell growth. The HER2 is considered as an orphan receptor because it needs no ligand to form homodimers, thereby contributing significantly to uncontrolled intracellular signaling. Heterodimers are also formed in some cases.

We present a method to study the distribution patterns of proteins in the plasma membranes of intact cells in liquid, which we successfully applied study HER2 and EGFR on SKBR3 breast cancer cells [1, 2]. The proteins were labeled with fluorescent quantum dots (QDs), exhibiting a core of an electron dense material. In order to detect the receptors without perturbing the native equilibrium, a short peptide was used for specific binding of the streptavidin-biotin coupled QDs [3]. The cells were fixed immediately after the labeling but kept in their native liquid state and staining was avoided. The overall cellular distributions were first evaluated using direct interference contrast (DIC) light microscopy, and fluorescence microscopy. These images provided an overview of the membrane topography, and the average amount of receptor in the membrane. Selected suitable regions were then studied at the nanoscale with environmental scanning electron microscopy (ESEM), while keeping the cells in their hydrated state (Fig. 1). The microscope was equipped with a scanning transmission electron microscopy (STEM) detector, so that strong contrast was obtained on the QDs (Fig. 2). Thousands of label positions were automatically detected and statistically analyzed via the calculation of the pair correlation function. The analysis results differentiated between distinct functional cellular regions.

Conventional biochemical methods are capable of studying the distribution of the receptors in protein complexes from pooled cellular material but miss information about expression levels at the cellular level. In particular, cancer cells are known to exhibit significant differences in the expression level of the HER2 receptor between individual cells and even between distinct functional membrane regions, representing the characteristic feature of heterogeneity derived from the accumulation of various genetic alterations. Optical methods, on the other hand, do not exhibit sufficient spatial resolution to resolve the individual constituents of proteins complexes, and only indirect information is obtained about the proximity of proteins. A particular problem is the inevitable random occurrence of proteins in close proximity in relevant regions with a high protein density. Our method is capable of distinguishing between random occurring distances and preferred distances indicating the actual presence of dimers or higher order complexes, because the distances can be directly measured with $3 \mathrm{~nm}$ resolution. 
References:

[1] D.B. Peckys and N. de Jonge, Microsc. Microanal. 20 (2014), p. 346.

[2] D.B. Peckys et al , Sci. Rep. 3 (2013), p. 2626-1.

[3] D.B. Peckys, V. Bandmann and N. de Jonge, Meth. Cell Biol. 124 (2014), p. 305.

[4] We thank E. Arzt for his support through INM. Research in part supported by the Leibniz Competition 2014.

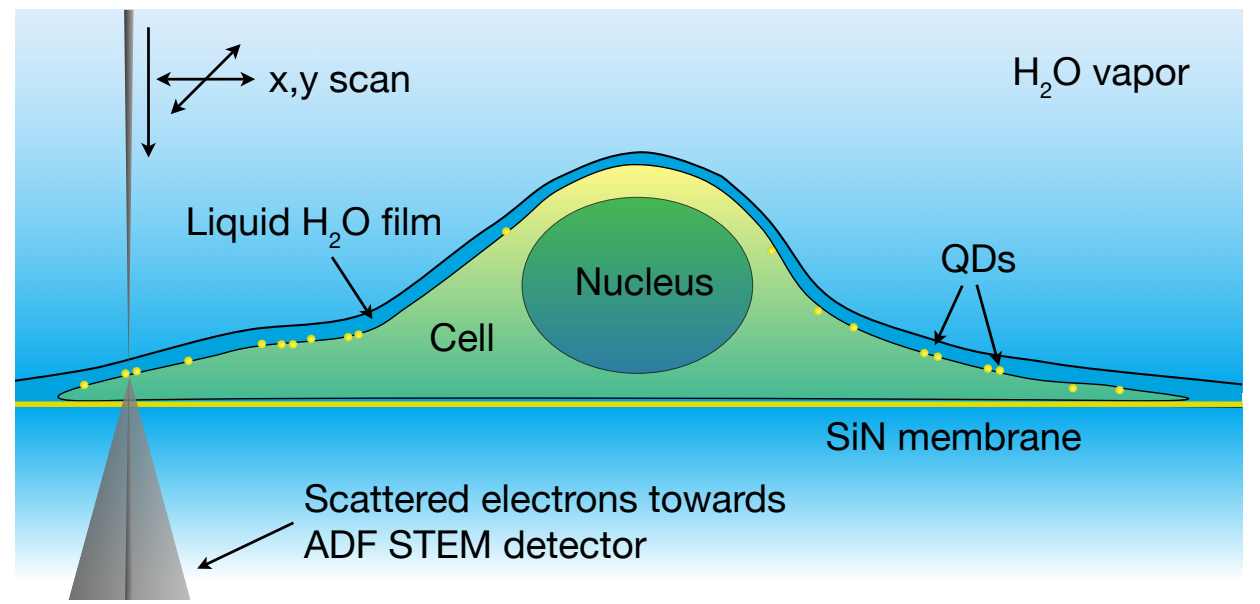

Figure 1. A cell grown on a silicon nitride ( $\mathrm{SiN})$ membrane containing proteins labeled with QDs. A liquid water film is maintained over the cell, while the sample is surrounded by a water vapor environment. The ESEM electron beam scans the sample, and contrast is obtained using the annular dark field (ADF) STEM detector located underneath the sample.

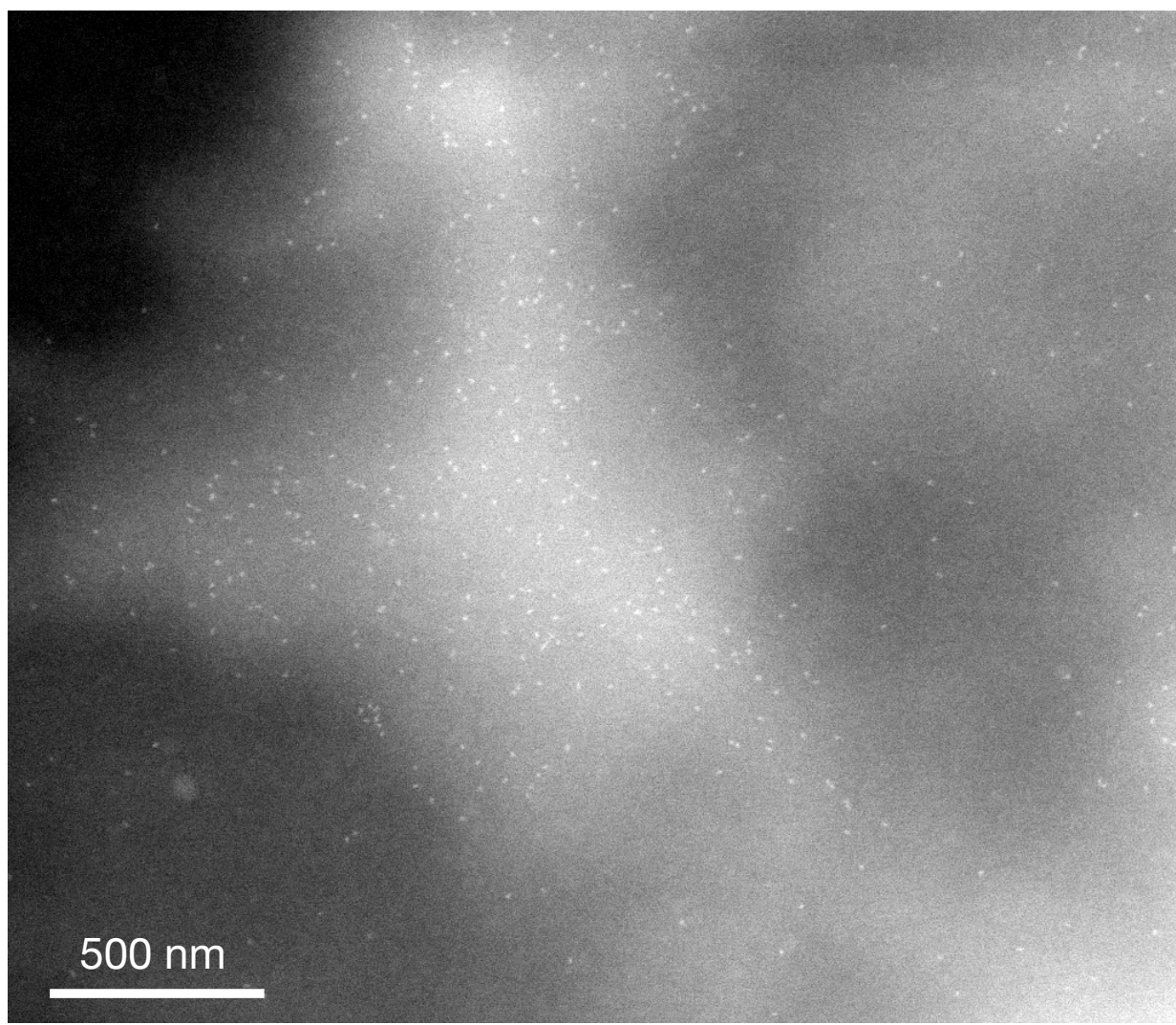

Figure 2. ESEM micrograph showing a membrane area of an intact SKBR3 breast cancer cell in liquid state. The white spots indicate positions of individual QD-labeled HER2 proteins. Many homodimers and large order clusters are visible. The image was recorded using an electron energy of $30 \mathrm{keV}$, at a vapor pressure of $740 \mathrm{pA}$ and a temperature of $3.0^{\circ} \mathrm{C}$. The image acquisition time amounted to $72 \mathrm{~s}$. 